\title{
The Influence Of Orthodontic Treatment On Dental Pulp Response To Sensitivity Tests
}

\author{
Ramona Vlad, DMD \\ Department of Odontology and Oral Pathology, Faculty of Dental Medicine, \\ University of Medicine and Pharmacy of Tîrgu Mureș, Romania \\ Irinel Panainte, DMD \\ Department of Orthodontics, Faculty of Dental Medicine, \\ University of Medicine and Pharmacy of Tîrgu Mureș, Romania \\ Liana Hantoiu, Lecturer, DMD, PhD \\ Department of Prosthetic, Dentistry Faculty of Dental Medicine, \\ University of Medicine and Pharmacy of Tîrgu Mureș, Romania \\ Monica Monea, Associate Professor, DMD, PhD \\ Department of Odontology and Oral Pathology, Faculty of Dental Medicine, \\ University of Medicine and Pharmacy of Tîrgu Mureș, Romania
}

doi: 10.19044/esj.2016.v12n6p322 URL:http://dx.doi.org/10.19044/esj.2016.v12n6p322

\begin{abstract}
Aim of our study. To evaluate the difference between sensory detection threshold reactions of teeth under orthodontic treatment with fixed appliances, determined by electrical and thermal pulp testing. Material and methods. After a selection based on inclusion and exclusion criteria, 51 patients with a mean age of 19,3+/- 3,6 years old who presented to the Center for Integrated Dental Medicine of the Faculty of Dental Medicine from Târgu Mureș for orthodontic treatment had been included in this study. The reactions to thermal and electrical pulp testing were measured at baseline, after 8 weeks of treatment and during the contention period. Results. The application of orthodontic force immediately increased the response threshold which peaked after 8 weeks. Conclusions. Based on these results we conclude that dental specialists should interpret with caution the results of electric pulp testing in teeth under orthodontic treatment with fixed appliances as the cold tests with ethyl chloride are more reliable.
\end{abstract}

Keywords: Orthodontic treatment, vitality testing, dental pulp

\section{Introduction}

The screening of tooth vitality is recommended before any orthodontic or major restorative procedure in order to determine whether the 
dental pulp is vital or not and it is usually carried out by electrical (EPT) and thermal stimulation. An ideal pulp tester should provide: a simple, objective, standardized, reproducible, non-painful, non-injurious, accurate and inexpensive way of assessing the exact condition of the pulp tissues at any given time (Chambers 1985). These are simple and non-invasive methods which offer important clinical information regarding the health status of the pulp tissue, as morphological alterations of cells and dental nerve fibers are accompanied by abnormal responses to sensitivity tests (Modaresi et al 2015, Lin et al 2008). After a force is applied on a tooth, an alteration of the physiological status of periodontal and pulp tissue may develop, with consequences on the response to electrical or thermal sensitivity tests. These results are not always reliable, therefore a correlation with other symptoms and clinical findings is mandatory in order to have a correct diagnosis (Hall et al 2008, Javed et al 2015). The aim of our prospective study is to evaluate the difference between sensory detection threshold response by electric and thermal pulp testing of teeth under orthodontic treatment with fixed appliances, before and after the brackets and arch wires were fixed, compared to the results obtained in the contention phase.

\section{Material and methods}

Our study was conducted in the Center for Integrated Dental Medicine (CIDM) of the University of Medicine and Pharmacy of Tîrgu Mureș, Romania between 2012 and 2015. After the research protocol was approved by the Ethics Committee of our university, a total of 276 teeth in 61 patients, 38 female and 23 men with a mean age of 19,3+/- 3,6 years old who presented for orthodontic treatment had been selected and included in this study, based on a written consent signed by all participants. The inclusion criteria were: cases with class I malocclusion and moderate crowding, the need for fixed orthodontic treatment, no systemic disease, positive reactions to sensitivity tests for maxillary front teeth. The exclusion criteria were applied to patients with a history of recent teeth trauma, caries, restorations, periodontal lesions or teeth partially erupted. All patients were subjected to dental examination, panoramic radiographic and periodontal evaluation and sensitivity tests were applied on maxillary front teeth at three treatment appointments: baseline (before the appliance was fixed), after 8 weeks of treatment and during the contention phase (8-10 weeks after the brackets and arch wires were removed). The teeth were isolated with cotton rolls and dried with compressed air. During the procedure for the cold test a cotton pellet with ethyl chloride handled with a dental pliers was placed in the incisal third of the vestibular surface, away from the orthodontic elements and gingival margin. For EPT the electrode of the device (Parkell, Farmigdale, NY, USA) was moist in tooth paste and placed in the same 
manner. The patients were asked to indicate the presence of pain to thermal test by raising their right hand which was recorded as positive if the reaction occurred in the first 5 seconds after application; the readings from the display of the instrument for EPT evaluation were also recorded. All observations were made by the same operator using the same instruments. The results were evaluated using the Graph Pad demo version software. The collected data were analyzed and the non-parametric qualitative chi-square test was used in order to determine the differences between measurements obtained at different time intervals. The level of statistical significance was set at 0.05 (p $<0.05)$.

\section{Results}

All teeth tested (107 canines, 94 central incisors and 75 lateral incisors) gave positive responses to thermal stimuli and EPT prior to orthodontic treatment and these recordings were considered baseline. The results showed that 8 weeks after the application of an orthodontic force there was an increased threshold to both EPT and thermal testing, with more teeth giving negative response to electric stimulation. From the total 276 teeth included in the study, 34\% failed to respond to EPT and 26\% to thermal testing with ethyl chloride. The differences between measurements done at baseline and after 8 weeks from the application of orthodontic forces were statistically significant (Table 1).

Table1. Results of sensitivity tests recorded after 8 weeks of treatment compared to baseline

Method/ Time

EPT

(mean +/- SD)

Cold test
Baseline

$2,81+/-0,80$

$100 \%$ positive

*statistically significant differences
After 8 weeks

$6,72+/-0,26$

$74 \%$ positive

$p$ value

$\mathrm{p}<0,05^{*}$

$\mathrm{p}<0,05^{*}$

In order to evaluate if the high stimulation threshold for EPT and negative responses for thermal test represent permanent modification in teeth under orthodontic treatment we compared the baseline readings to those measured during the contention phase (Table 2).

Table2. Results of sensitivity tests recorded during contention phase compared to baseline

Method/ Time

EPT

(mean +/- SD)

Cold test
Baseline

2,81+/- 0,80

100\% positive
Contention

3,41+/- 0,68

93\% positive $p$ value

$\mathrm{p}>0,05$

$\mathrm{p}>0,05$

The responses to electrical stimulation demonstrated a higher threshold but there was no statistically significant difference with baseline values. The same was noted for thermal stimulation, where 93\% of teeth gave a positive response to cold within 5 seconds after the stimulus was applied on the vestibular surface. 


\section{Discussion}

The most common pulp testing method used by dental practitioners is to record the response of dental pulp to thermal stimuli. A positive reaction response to cold usually indicated a vital pulp, regardless if the dental pulp is healthy or not and ideally the result should be compared to electric pulp testing (Gopikrishna et al 2009). The electric test is by many considered to be the least reliable method but as it has many advantages and therefore must be part of the armamentarium of each dental office. The main advantage of the electric pulp testing is that a specific value is obtained for the reaction threshold of the tooth, which can be used for comparison between different teeth or the same tooth over time (Lin et al 2008). In our study we used thermal and electrical pulp testing for teeth subjected to orthodontic treatment with fixed appliances and the results showed that short after the forces were applied there was an increase in threshold for electric stimulation, which is in accordance with the observations of other studies (Modaresi et al 2015, Javed et al 2015, Cave et al 2002, Alomari et al 2011 ).

Thermal stimulation may be of value in provoking symptoms for teeth under orthodontic treatment, after trauma or with asymptomatic pulpitis. At the time of examination, the patient may present with no pain and no signs or symptoms of dental pulp alteration. Both cold and heat tests can be used, although in most of the cases heat proved to be the most effective. Both unmyelinated and myelinated fibres transmit stimuli and the sensory detection threshold response of the dental pulp to electrical stimulus originated through the myelinated A-delta fibres located in the subodontoblastic layer. These fibres have been identified as having characteristics of a low sensory threshold and fast conduction of sharp pain. They are responsible for dentine sensitivity. A physiologic change in the dental pulp can affect the integrity of A-fibres and as a consequence, the response thresholds to electrical stimulation may increase or it may fail to initiate any response at all. The slow conducting unmyelinated C-fibres are responsible for the poorly localized sensations associated with pulpal injury. The degenerative changes may manifest long after the time of injury but some pathologic signs were observed also right after the force was applied. The tissue changes are reversible if the aggression does not exceed the threshold of tissue tolerance (Ferreira et al 2013). There is evidence suggesting that changes in blood flow can modulate sensory nerve function and a reduction in pulpal blood flow can suppress the activity of the A-fibres. The nerve activity could also be influenced by inflammatory mediators and the anatomical maturation of the tooth, as immature teeth might give negative results to EPT. Therefore, it is important that all teeth that had been under fixed orthodontic treatment and gave negative responses to sensitivity 
testing to be periodically examined in order to identify as early as possible the need for endodontic treatment.

\section{Conclusion}

Within the limitation of our study we can conclude that teeth submitted to orthodontic movement present a higher incidence of negative sensitivity during the early treatment stages. The results of our study suggest that dental specialists should interpret with caution the results of electric pulp testing in these clinical situations, as the cold tests with ethyl chloride are more reliable.

\section{References:}

Chambers I.G. The role and methods of pulp testing in oral diagnosis: a review. Int Endod J; 1982 (15): 1-5.

Modaresi J., Aghili H., Dianat O., Younessian F., Mahjour F. The effect of orthodontic forces on tooth response to electric pulp test. Iran Endod J; 2015 (10): 244-247.

Lin J., Chandler N. Electric pulp testing: a review. Int Endod J; 2008 (41): 365-374.

Hall C.J., Freer T.J. The effects of early orthodontic force application on pulp test responses. Aust Dent J; 1998 (43): 359-361.

Javed F., Al-Kheraif A.A., Romanos E.B., Romanos G.E. Influence of orthodontic forces of human dental pulp: A systematic review. Arch Oral Biol; 2015 (60): 347-356.

Gopikrishna V., Pradeep G., Venkateshbabu N. Assessment of pulp vitality: a review. Int J of Paediatric Dentistry; 2009 (19): 3-15.

Cave S., Freer T., Podlich H. Pulp-test responses in orthodontic patients. Aust Orthod J; 2002 (18): 27-34.

Alomari F.A., Al-Habahbeh R., Alsakarna B.K. Responses of pulp sensibility tests during orthodontic treatment and retention. Int Endod J; 2011(44): 635-643.

Ferreira L.L., Filho E.M., Rodrigues C.L., da Silveira Bueno C.E., Cunha R.S. Lack of pulp sensitivity in maxillary canines submitted to orthodontic traction: a retrospective clinical study. RSBO; 2013(10): 29-33.

Corresponding author: Monica Monea, Associate Professor, University of Medicine and Pharmacy of Tîrgu Mureș, Faculty of Dental Medicine, Gheorghe Marinescu Street, No. 38, 540139, Tîrgu Mureș, Romania 